\title{
ANÁLISIS DE RIESGO DE ACCIDENTALIDAD DE VEHICULOS PESADOS EN LA VÍA CÚCUTA - PAMPLONA
}

\section{RISK ANALYSIS OF HEAVY VEHICLE ACCIDENTS ON THE CÚCUTA - PAMPLONA ROAD}

\author{
Ing. Adrián Ricardo Patiño Morantes*, PhD. Gonzalo Guillermo Moreno \\ Contreras", PhD. Bladimir Azdrúbal Ramón Valencia*
}

"Universidad de Pamplona, Facultad de Ingenierías y Arquitectura, Grupo de Investigación en Ingeniería Mecánica Universidad de Pamplona (GIMUP), Km 1 Vía

Bucaramanga Ciudad Universitaria, Pamplona, Norte de Santander, Colombia. Tel.: (+57 7) 568 5303, Fax: (+57 7) 568 5303, Ext. 144

E-mail: adrian_ricardo_19@hotmail.com, gmoren,hbladimir@unipamplona.edu.co

Resumen: El presente artículo muestra en detalle la metodología empleada para determinar los puntos críticos de accidentalidad de vehículos pesados en la vía Cúcuta Pamplona. Esta vía es muy importante, ya que conecta el nororiente colombiano con el resto del país, por donde se mueven pasajeros y gran parte del comercio internacional con Venezuela. A través de una encuesta dirigida a los usuarios de la vía, se pudo determinar los puntos críticos de accidentalidad, los tipos de accidentes más frecuentes y la clase de vehículos pesados con mayor incidencia de accidentalidad.

Palabras clave: Accidentalidad, vehículos pesados, puntos críticos, vía.

\begin{abstract}
This article shows in detail the methodology used to determine the critical points of heavy vehicle accidents on the Cúcuta - Pamplona road. This route is very important, since it connects the northeast of Colombia with the rest of the country, where passengers and much of the international trade with Venezuela go through. Through a survey addressed to road users, it was possible to determine the critical accident points, the most frequent types of accidents and the class of heavy vehicles with the highest incidence of accidents.
\end{abstract}

Keywords: Accident, heavy vehicles, critical points, road.

\section{INTRODUCCIÓN}

En la actualidad para los países es de vital importancia el transporte de mercancías, lo cual incluye tanto su producción como los productos que importa, este transporte en su mayoría se realiza a través de vehículos pesados, todo esto permite impulsar gran parte del crecimiento económico y social de un país. Según García et al., (2012), a través de los años, estos vehículos han tenido mejoras significativas con respecto a su velocidad, capacidad de carga, comodidad entre otros aspectos. Todo esto ha permitido el aumento de vehículos en las vías y por consiguiente también el aumento del número de accidentes y la disminución de la seguridad vial.

De acuerdo con Moreno et al., (2018), los accidentes más frecuentes de los vehículos pesados cuando realizan curvas son el volcamiento y la salida de la pista (deslizamiento), los cuales son problemas relacionados con la estabilidad de estos vehículos, en este sentido, el Static Rollover Threshold (SRT) por sus siglas en inglés, es un factor que permite determinar qué tan seguro es un vehículo en términos de estabilidad y a qué velocidad puede transitar por determinada curva (Moreno et al., 2018a). 
Teniendo en cuenta este aspecto, la seguridad vial está ligada directamente con tres factores: el conductor, el vehículo y la carretera, relacionados entre sí y en la forma en que ellos actúan y como se desenvuelvan, se observa el aumento o la disminución de la seguridad vial.

Por consiguiente, la seguridad vial en esta vía es de vital importancia, ya que es la principal ruta de comunicación del Departamento Norte de Santander con el resto del país y siendo adicionalmente la vía por donde se mueve gran parte del comercio internacional con Venezuela.

Teniendo en cuenta todos estos aspectos, el presente trabajo se desarrolló, con la finalidad de dar a conocer a los usuarios de la vía, en que trayectos o tramos de esta vía deben transitar con mayor precaución para evitar accidentes, y además, porque a través de los años ha habido ciertos puntos críticos de accidentalidad que no han podido ser disminuidos o eliminados en su totalidad, debido a muchos factores como la falta de señalización, alta velocidad de los vehículos, el mal estado de la vía entre otros.

\section{ANTECEDENTES}

Usualmente los vehículos pesados no representan gran número de accidentes por volcadura o salida de la vía, pero debido a la gravedad de estos accidentes cuando se presentan, es un tema importante para ser analizado con respecto a otros accidentes como lo son los choques, atropellos e incendios de vehículos (Güemez, 2013; Jabour, 2010; Moreno et al., 2017).

De acuerdo con Grislis (2010), la accidentalidad por problemas relacionados con la estabilidad de estos vehículos se ha incrementado debido a: mal estado de la infraestructura vial, falta o mala señalización, altas velocidades al tomar una curva, imprudencias de los conductores al realizar diferentes maniobras y al aumento de vehículos en las vías.

De acuerdo con el informe de la Comunidad Andina de Naciones (CAN, 2016) en Colombia en el año 2015, se presentaron 8484 accidentes por volcamiento de vehículos, siendo el $77 \%$ de estos accidentes en la zona rural, representada por las vías primarias nacionales.

En la actualidad, la accidentalidad por volcamiento de vehículos pesados está relacionada con la estabilidad y esta a su vez con la altura de su centro de gravedad (Chu et al., 2018), que en comparación con otros vehículos es elevada. Para disminuir esta accidentalidad y mejorar la seguridad vial, se han diseñado una serie de dispositivos viales y mecánicos que incluyen:

1. Señales de tránsito que indican a qué velocidad deben tomar la curva estos vehículos. Estas señales presentan una desventaja, ya que no se diseñan teniendo en cuenta parámetros del vehículo, geometrías de la vía o condiciones climáticas.

2. Sistemas de estabilidad del vehículo, que le permiten al conductor disminuir la velocidad del vehículo y evitar el volcamiento. Además, permite controlar de forma directa la seguridad, el confort y brindar mejores condiciones de maniobrabilidad al momento de acelerar, frenar y girar el vehículo (Navarro et al., 2018).

\section{ANÁLISIS DE RIESGO}

La accidentalidad de vehículos pesados en relación con volcamiento y salida de la vía en la vía Cúcuta - Pamplona (Ruta 55), entre los años 2010 y lo que va corrido del 2018 es de 25 accidentes, según datos aportados por la Policía de Tránsito y Transporte, seccional Norte de Santander, Colombia (Policía Nacional 2018). De acuerdo con los relatos aportados, en estos accidentes se observan consecuencias como muertos, heridos, daño de los vehiculó, perdidas de mercancía y cierres de la vía, pero no hay cifras concretas o exactas de cada uno de estos efectos.

Adicionalmente, se puede manifestar que en estos accidentes, las causas probables fueron: exceso de velocidad del vehículo, falta de pericia del conductor, fallas mecánicas, adelantar en curva, o cansancio del conductor.

Según los datos aportados, los puntos críticos de accidentalidad de vehículos pesados en la vía Cúcuta - Pamplona son:

> Kilómetro 71+280 denominado la curva de los Adioses,

> Kilómetro 84+300 denominado las S antes de las Cocalinas,

> Kilómetro 85+450 denominado las Cocalinas,

> Kilómetro 98+060 denominado el Diamante,

> Kilómetro 109+600 denominado la Donjuana.

El kilómetro característico de cada punto crítico es tomado con referencia de la vía Presidente - 
Pamplona - Cúcuta, donde Presidente (vía Málaga, Santander) es el kilómetro 00+000, la entrada a Pamplona es el kilómetro 70+200 y Cúcuta es el kilómetro 131+500 (Policía Nacional, 2018).

Sin embargo, y debido a que muchas veces los accidentes no son reportados o no se tiene registro de ellos, no se cuenta con una estadistica detallada que permita identificar los puntos de mayor riesgo para los vehiculos pesados.

Con el fin de obtener información más detallada sobre la accidentalidad de la vía, se realizó una encuesta de accidentalidad de vehículos pesados en la vía Cúcuta-Pamplona, aplicada a usuarios frecuentes de la vía (Tabla 1), los cuales conocen de primera mano los pormenores de esta.

Tabla 1. Usuarios de la vía.

\begin{tabular}{cc}
\hline Personas encuestadas & Cantidad \\
\hline $\begin{array}{c}\text { Conductores de vehículos } \\
\text { pesados }\end{array}$ & 40 \\
Conductores de servicio público & 40 \\
Personas que viven o trabajan en & 20 \\
la vía & \\
Total encuestados & 100 \\
\hline
\end{tabular}

La primera pregunta de esta encuesta indaga sobre el tipo de accidente más frecuente de los vehículos pesados que transitan esta vía, la Fig. 1 muestra que los accidentes relacionados con la estabilidad de los vehículos pesados como lo son el volcamiento y la salida de la pista son los más frecuentes.

\section{TIPO DE ACCIDENTE}

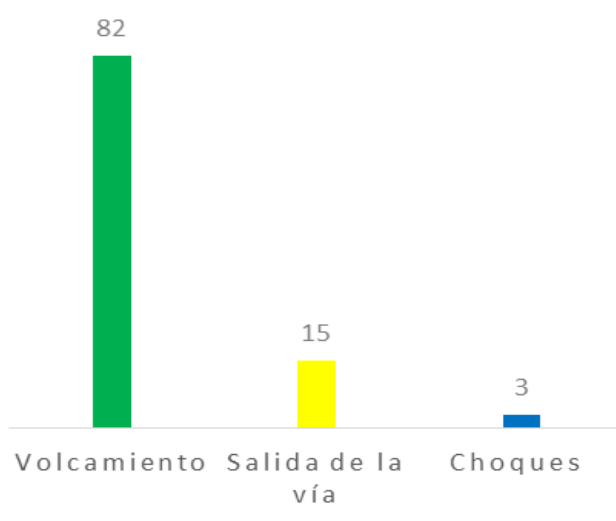

Figura 1. Tipos de accidentes más frecuentes en la vía Cúcuta - Pamplona.

Otro aspecto importante en la presente encuesta fue detallar los puntos críticos de la vía, se determinaron nueve puntos críticos con riesgo de accidentalidad por volcamiento y salida de la vía (Fig. 2), siendo los puntos críticos con mayor peligrosidad para los usuarios de la vía, Las $\mathrm{S}$ de Corozal, La entrada a Pamplonita, El Diamante, las Cocalinas, de los cuales algunos coinciden con los indicados por la Policía de Tránsito y Transporte y hay otros que también representan especial cuidado como:

Kilómetro 81+000, denominado La Entrada a Pamplonita,

$>$ Kilómetro 84+550, denominado El Rincón de los Caballos,

> Kilómetro 87+220, denominado La Miguelera,

$>$ Kilómetro 124+100, denominado las $\mathrm{S}$ de Corozal,

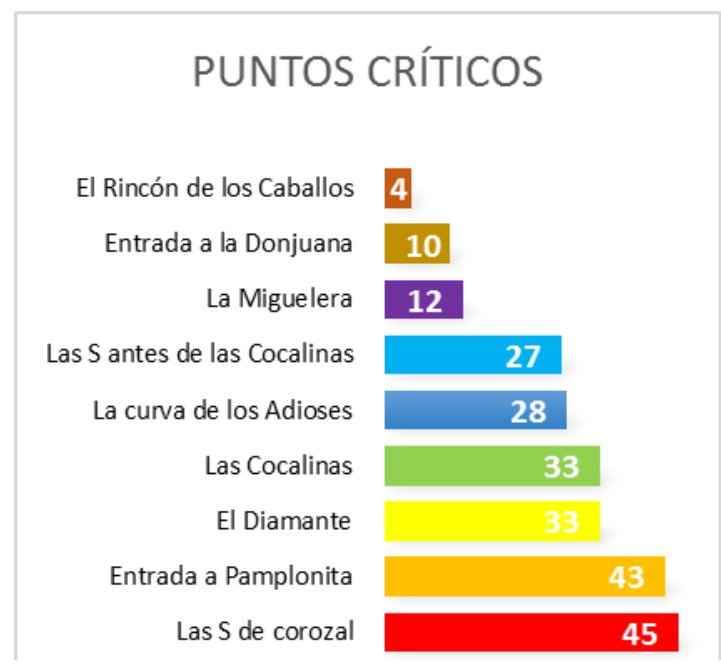

Figura 2. Puntos críticos de accidentalidad de la vía Cúcuta - Pamplona.

De acuerdo con los usuarios de la vía, estos trayectos requieren de especial cuidado por parte de los conductores, ya que se requiere de disminuciones altas de velocidad en pequeños trayectos y alta pericia de los conductores al transitar por ellos. Estos aspectos son más críticos cuando por estas vías transitan conductores nuevos, los cuales argumentan que la señalización vial no es la adecuada para la peligrosidad que estos puntos representan en relación a este tipo de vehículos.

Otro aspecto importante para el presente trabajo fue determinar, es el tipo de vehículos pesados de carga con mayor incidencia o probabilidad de accidentes de la vía Cúcuta - Pamplona, al respecto se observó que el vehículo más propenso a los 
accidentes es el tracto-camión, como se describe en la Tabla 2.

Tabla 2. Tipo de vehículos pesados que presenta mayor accidentalidad.

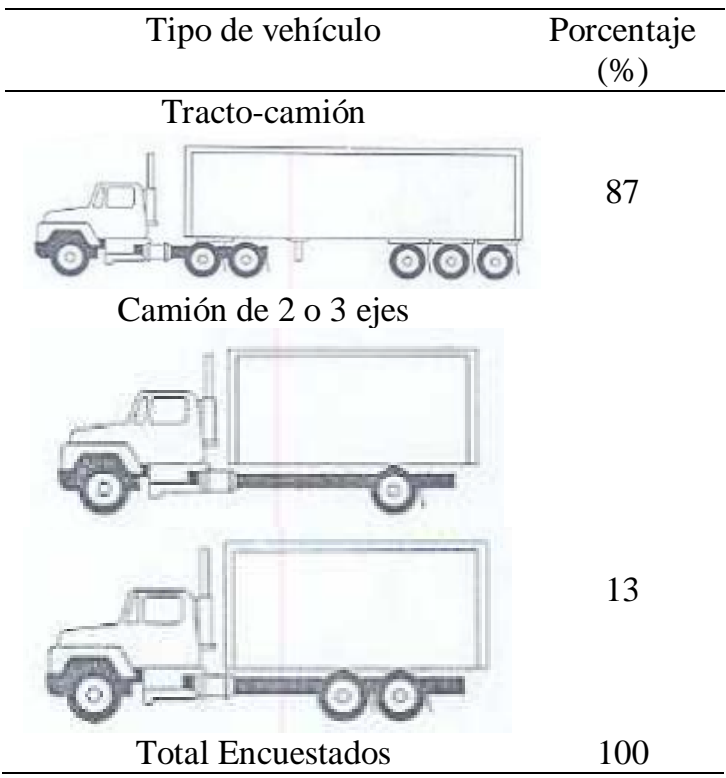

Cabe resaltar, que los vehículos tipo Tracto-camión son más propensos al volcamiento por ser articulados, más largos, y más pesados en relación con los otros vehículos. Otros factores que influyen en el vuelco de estos vehículos son: las condiciones geométricas de la vía, el desempeño del vehículo y el comportamiento o maniobrabilidad del conductor Grislis (2010).

Posterior a ello, se pretendía encontrar que factores de riesgo tienen mayor influencia en la accidentalidad de vehículos pesados, y se observó que la alta velocidad para tomar una curva es la que presentaba mayor frecuencia como lo describe la Fig. 3.

Los encuestadores afirmaban que las altas velocidades se debían a la señalización incorrecta en estos trayectos, lo que conlleva tomar estas curvas sin mayor precaución y su vez ocasionar accidentes. También deducían, que en la mayoría de los casos la alta velocidad iba acompañada de maniobras peligrosas del conductor cuando desconocía la vía e intenta evitar los accidentes.

Otro factor determinante es la falta de pericia del conductor al afrontar cada punto crítico, debido a que estos vehículos requieren un mayor espacio para transitar por su longitud y pueden generar molestias a otros usuarios de la vía Güemez (2013).

\section{FACTORES DE RIESGO}

\begin{tabular}{r|c} 
Mal diseño de la vía & 1 \\
Mala señalización de la vía & 2 \\
Condiciones de carga del vehículo & 3 \\
Mala superficie de la vía & 3 \\
Falta de pericia del conductor & 9 \\
Maniobras peligrosas conductor & \\
Alta velocidad &
\end{tabular}

Figura 3. Factores de riesgo que influyen en la accidentalidad de vehículos pesados.

Otro factor importante a la hora de estudiar los accidentes de tránsito son las causas que lo producen, ya que de esta forma se pueden buscar soluciones que nos ayuden a dismunirlos o eliminarlos.

Según García et al., (2012), estas causas pueden ser analizadas con tres momentos fundamentales: problemas evidentes que requieren un estudio superficial, problemas semiprofundos que necesitan un poco mas de investigación y los problemas profundos en donde se evalua desde el más minimo detalle para encontrar su causa final.

Cuando se inicío la busqueda de la información de accidentalidad en esta vía, se observó que todos los accidentes son tratados como estudio superficial, es decir, que su investigación no era a fondo, lo que conlleva al desconocimiento de sus causas reales y a la falta de registros actualizados con su detallada información, por esta razón, se optó por desarrollar este estudio de accidentalidad.

Según Güemez (2013), uno de los aspectos que podrían estudiarse para esclarecer las causas del accidente es el estudio del comportamiento dinámico del vehículo, condiciones de este, condiciones de la via, entre otros.

En la actualidad los accidentes de vehículos pesados no son frecuentes en esta vía, pero son de especial interes las diferentes consecuencias o efectos que causan. En la Fig. 4, se observa que la principal consecuencia que presentan estos accidentes es la pérdida de mercancia, sin dejar de lado los heridos ni las perdidas humanas.

Las perdidas de la mercancia afectan económicamente a los propietarios de la carga transportada, los consumidores o clientes finales de 
los productos, los conductores de vehículos pesados que deben en algunas ocaciones que responder por los daños causados tanto del vehículo como de la carga transportada y por ultimo las empresas aseguradoras de los vehículos.

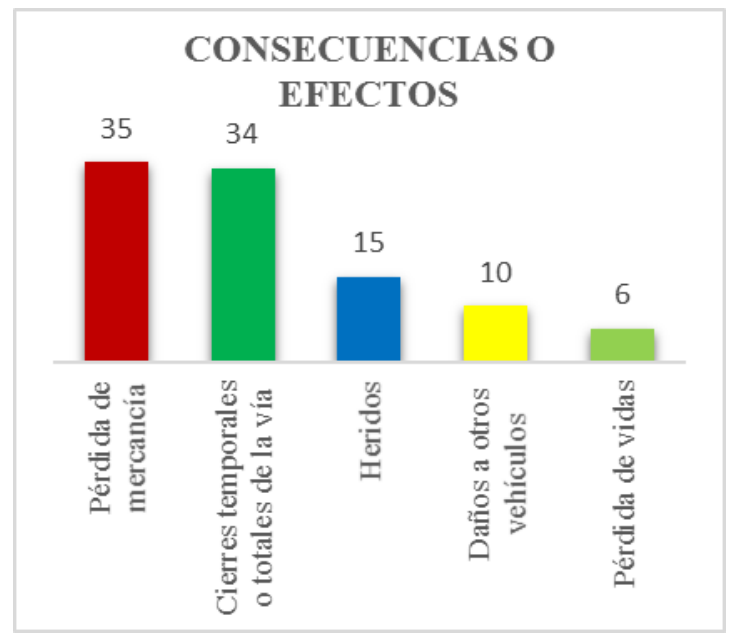

Figura 4. Consecuencias o efectos que producen los accidentes.

Otra consecuencia que afecta en gran proporción son los cierres temporales o totales de las vías, ya que perjudican la movilidad de los demás usuarios que transitan por la vía y a su vez retrasa los tiempos de llegada a sus destinos.

La pérdida de vidas es una consecuencia de gran importancia debido a que los accidentes de tránsito son uno de los principales responsables de los índices de mortalidad en Colombia según el Ministerio de Salud (MinSalud, 2015), ocupando el segundo lugar, con un porcentaje del $18,81 \%$ de la totalidad de las muertes en el año 2014. El primer puesto de mortalidad lo ocupan las agresiones (homicidios).

En algunos casos los accidentes pueden ocasionar daños a otros vehículos, que a su vez pueden causar heridos y muertos, esto disminuye la seguridad vial de las personas que transitan por la vía.

Grislis (2010) asegura que todas estas consecuencias ocasionadas por accidentes de vehículos pueden reducirse si:

1. Se conduce de forma adecuada respecto a las condiciones de carga, diseño geométrico de las carreteras, parámetros técnicos de los vehículos, condiciones climáticas y condiciones superficiales la vía.
2. Si todas las personas que transitan en la vía siguen a cabalidad las señales de tránsito y conducen con prudencia y precaución.

\section{CONCLUSIONES}

Al analizar los diferentes aspectos relacionados con el comportamiento de los vehículos pesados en la vía Cúcuta - Pamplona, se pudo deducir que existen problemas de seguridad vial relacionados con la accidentalidad por volcamiento y salida de la vía de estos vehículos. Se encontraron nueve puntos críticos de accidentalidad y se observó que estos accidentes se presentan debido a: las altas velocidades para tomar una curva, falta de pericia y maniobras peligrosas del conductor asociadas con el mal estado de la vía, la falta de demarcación en algunos trayectos y la carencia de señalización vial cuando los conductores no conocen la vía.

Estos accidentes traen consigo efectos secundarios como: cierres temporales o totales de la vías, pérdida de mercancías, heridos, muertos y daños a otros vehículos, que pueden afectar directa o indirectamente a los usuarios de la vía y a la comunidad nortesantandereana ya que estos vehículos representan la mayor parte del transporte de mercancías de la región.

También se determinó que el vehículo más propenso a accidentalidad por volcamiento es el tracto-camión, debido a que son articulados, más largos y más pesados que la mayoría de los vehículos, lo cual requiere una mayor maniobrabilidad por parte del conductor y más espacio para transitar con referencia al vehículo.

\section{REFERENCIAS}

Alvernia Acevedo, S., \& Rico Bautista, D. (2017). Análisis de una red en un entorno IPV6: una mirada desde las intrusiones de red y el modelo TCP/IP. REVISTA COLOMBIANA DE TECNOLOGÍAS DE AVANZADA, 1(29).

CAN. (2016) Accidentes de tránsito en la Comunidad Andina de Naciones..

Chu, D., Li, Z., Wang, J., Wu, C., \& Hu, Z. (2018). Rollover speed prediction on curves for heavy vehicles using mobile smartphone Measurement, 130, 404-411.

García Depestre, R. A., Delgado Martínez, D. E., Díaz García, E. E., \& García Armenteros, R. R. (2012). Caracterización de la accidentalidad vehicular y análisis de las 
causas en la provincia de Villa Clara, Cuba. Dyna, 79(175).

Grislis, A. (2010). Longer combination vehicles and road safety. Transport, 25(3), 336343.

Güemez Shedden, C. R. (2013). Comportamiento dinámico de los vehículos de carga pesada en el cantón de San José (2011-2012). Infraestructura Vial Vol. 15 Núm. 26.

Jabour, C. C. (2010). Custos dos Acidentes nas rodovias federais, avaliando seus impactos sócio-econômicos para o país e efetuando sua aplicação na engenharia rodoviária.

Moreno, G. G., Florez, E. G., Peña, C. A. (2017) stability study of heavy vehicles, Revista Colombiana de Tecnologías de Avanzada, ISSN 1692-7257. 2017. v. 2 (No 30). pp. 1-6.

Moreno, G. G., Vieira, R. S., Martins, D. (2018). Highway designs: effects of heavy vehicles stability. Revista DYNA, ISSN 0012-7353. 2018. v. 85 (No 205). pp. 205-210.

DOI: 10.15446/dyna.v85n205.69676

Moreno, G. G., Nicolazzi, L., Vieira, R. S., Martins, D. (2018a). Stability of Long Combination Vehicles. International Journal of Heavy vehicle Systems, ISSN online: 1741-5152. ISSN print: 1744232X. v. 25 (No 1). (2018) pp. 113-131. DOI: $10.1504 /$ IJHVS.2018.10011111

MinSalud, M. D. (2015). Mortalidad y lesiones por accidentes de transporte en Colombia, 2013-2014. 1-39.

Navarro, L., García, J. G., Rodríguez, H. R. (2018) cinematic and dynamic considerations for the development of control a un suspension system, Revista Colombiana de Tecnologías de Avanzada, ISSN 16927257. 2017. v. 1 (No 31). pp. 1-4.

Policía Nacional, (2018), Accidentes de Tránsito, Vía Cúcuta - Pamplona, Policía Nacional, Dirección de Tránsito y Transporte, Seccional Norte de Santander, Colombia, 2018, 1-2

S. A. A. Acevedo, D. R. Bautista. (2017). Análisis de una red en un entorno IPV6: una mirada desde las intrusiones de red y el modelo TCP/IP. REVISTA COLOMBIANA DE TECNOLOGÍAS DE AVANZADA, ISSN: 1692-7257. 1(29). 